\title{
Improve the dosimetric outcome in bilateral head and neck cancer (HNC) treatment using spot-scanning proton arc (SPArc) therapy: a feasibility study
}

Gang Liu ${ }^{1,2,3}$, Xiaoqiang Li $i^{2}$ An Qin², Weili Zheng², Di Yan², Sheng Zhang ${ }^{1}$, Craig Stevens², Peyman Kabolizadeh ${ }^{2 \dagger}$ and Xuanfeng Ding ${ }^{2^{*+}}$

\begin{abstract}
Background: To explore the dosimetric improvement, delivery efficiency, and plan robustness for bilateral head and neck cancer (HNC) treatment utilizing a novel proton therapy technique - the spot-scanning proton arc (SPArc) therapy.

Methods: We evaluated fourteen bilateral HNC patients retrospectively. Both SPArc and 3-field Intensity Modulated Proton Therapy (IMPT) plans were generated for each patient using the same robust optimization parameters. The prescription doses were 70Gy (relative biological effectiveness (RBE) for CTV_high and 60Gy[RBE] for CTV_low. Clinically significant dosimetric parameters were extracted and compared. Root-mean-square deviation dose (RMSDs) Volume Histogram(RVH) was used to evaluate the plan robustness. Total treatment delivery time was estimated based on the machine parameters.

Results: The SPArc plan was able to provide equivalent or better robust target coverage while showed significant dosimetric improvements over IMPT in most of the organs at risk (OARs). More specifically, it reduced the mean dose of the ipsilateral parotid, contralateral parotid, and oral cavity by $25.8 \%(p=0.001), 20.8 \%(p=0.001)$ and $20.3 \%(p=0.001)$ respectively compared to IMPT. This technique reduced D1 (the maximum dose covering 1\% volume of a structure) of cord and brain stem by $20.8 \%(p=0.009)$ and $10.7 \%(p=0.048)$, respectively. SPArc also reduced the average integral dose by $17.2 \%(p=0.001)$ and external V3Gy (the volume received 3Gy[RBE]) by $8.3 \%(p=0.008)$ as well. RVH analysis showed that the SPArc plans reduced the dose uncertainties in most OARs compared to IMPT, such as cord: $1.1 \pm 0.4 \mathrm{~Gy}[\mathrm{RBE}]$ vs $0.7 \pm 0.3 \mathrm{~Gy}[\mathrm{RBE}](p=0.001)$, brain stem: $0.9 \pm 0.7 \mathrm{~Gy}[\mathrm{RBE}]$ vs $0.7 \pm$ $0.7 \mathrm{~Gy}[\mathrm{RBE}](p=0.019)$, contralateral parotid: $2.5 \pm 0.5 \mathrm{~Gy}[\mathrm{RBE}]$ vs $2.2 \pm 0.6 \mathrm{~Gy}[\mathrm{RBE}](p=0.022)$ and ipsilateral parotid: $3.1 \pm 0.7 \mathrm{~Gy}[\mathrm{RBE}]$ vs $2.8 \pm 0.6 \mathrm{~Gy}[\mathrm{RBE}](p=0.004)$ respectively. The average total estimated treatment delivery time were $283.4 \pm 56.2 \mathrm{~s}, 469.2 \pm 62.0 \mathrm{~s}$ and $1294.9 \pm 106.7 \mathrm{~s}$ based on energy-layer-switching-time (ELST) of $0.1 \mathrm{~s}, 1 \mathrm{~s}$, and $5 \mathrm{~s}$ respectively for SPArc plans, compared to the respective values of $328.0 \pm 47.6 \mathrm{~s}(p=0.002), 434.1 \pm 52.0 \mathrm{~s}(\mathrm{p}=0.002)$, and $901.7 \pm 74.8 \mathrm{~s}(p=0.001)$ for 3 -field IMPT plans. The potential clinical benefit of utilizing SPArc will lead to a decrease in the mean probability of salivary flow dysfunction by $31.3 \%(p=0.001)$ compared with IMPT.

(Continued on next page)
\end{abstract}

\footnotetext{
* Correspondence: Xuanfeng.Ding@beaumont.org

†Peyman Kabolizadeh and Xuanfeng Ding shared senior authorship.

${ }^{2}$ Department of Radiation Oncology, Beaumont Health System, Royal Oak, MI 48074, USA

Full list of author information is available at the end of the article
}

(C) The Author(s). 2020 Open Access This article is distributed under the terms of the Creative Commons Attribution 4.0 International License (http://creativecommons.org/licenses/by/4.0/), which permits unrestricted use, distribution, and reproduction in any medium, provided you give appropriate credit to the original author(s) and the source, provide a link to the Creative Commons license, and indicate if changes were made. The Creative Commons Public Domain Dedication waiver (http://creativecommons.org/publicdomain/zero/1.0/) applies to the data made available in this article, unless otherwise stated. 
(Continued from previous page)

Conclusions: SPArc could significantly spare OARs while providing a similar or better robust target coverage compared with IMPT in the treatment of bilateral HNC. In the modern proton system with ELST less than $0.5 \mathrm{~S}$, SPArc could potentially be implemented in the routine clinic with a practical, achievable treatment delivery efficiency.

Keywords: Dosimetric outcome; bilateral head and neck cancer; spot-scanning; proton arc; delivery efficiency, Robustness

\section{Background}

The management of head and neck cancer (HNC) frequently involves radiation treatment. Previous studies have shown that external beam radiation therapy, including 3-dimensional conformal radiation therapy (3DCRT), intensity-modulated photon therapy (IMRT), and volumetric-modulated arc therapy (VMAT), improved HNC patient's outcome and quality of life significantly [1-3]. Currently, IMRT is the standard of treatment delivery for $\mathrm{HNC}$, given its ability to spare the adjacent organs-at-risk (OARs). On the other hand, proton beam therapy has been introduced to clinical practice to achieve better dose conformity and better OARs sparing such as spinal cord, brain stem, oral cavity, and parotid compared to IMRT or VMAT [4-6].

The recent development of Pencil Beam Scanning (PBS) technique, such as Intensity Modulated Proton Therapy (IMPT), utilizes numerous mono-energetic narrow beamlets ("spots") to paint the target volume layer by layer. Such a technique significantly improves the dose conformity over the traditional proton passivescattering technique. Although this active scanning delivery system provides the most considerable flexibility to shape the target volume dose distribution and pattern just like 3D printing in the human body, it is also highly susceptible to different uncertainties, including setup, range uncertainties [7-9], and especially geometric changes in bilateral HNC patients [10].

Due to the current technique limitation of proton treatment delivery efficiency, only a few beam angles are utilized for the treatment of bilateral $\operatorname{HNC}[11,12]$. Furthermore, the inherent characteristic of increased lateral penumbra due to the scattering may result in undesirable plan quality and conformity, which become very challenging to spare the critical structures adjacent treatment site using IMPT, such as the parotid and oral cavity in HNC [13, 14]. Besides, salivary flow dysfunction and xerostomia are the most common late side effects of radiotherapy (RT) for head-and-neck malignancies and a significant cause of decreased quality of life in survivors [15]. Thus, there is an immediate need to improve further the dosimetric treatment plan quality. A more recent study in 2018 found that with increased degrees of optimization freedom or more beam directions, the range shifter (RS) may not be needed in the treatment of bilateral HNC compared to the current standard-of-care approach, IMPT [12]. The study also found an interesting phenomenon that the dosimetric plan quality is getting better even without utilizing RS in bilateral HNC treatment. Unfortunately, such a proposal and approach with numerous beam angles may not be practically feasible in a proton therapy center due to prolonged treatment time. However, the idea of delivering the proton beam therapy through arc(s) trajectory with a continuously rotational gantry may overcome such limitation $[11,16]$. A new treatment technique, Spot-scanning Proton Arc therapy (SPArc) introduced by Ding et al. in 2016 could generate robust and delivery efficient proton arc therapy plans. This technique has the potential to be implemented into the existing clinical proton system $[17,18]$. To the best of our knowledge, this is the first comprehensive study to investigate the potential dosimetric and clinical benefits, delivery efficiency, delivery accuracy, and plan robustness of SPArc in the treatment of bilateral HNC.

\section{Methods}

\section{Treatment planning}

Fourteen bilateral HNC patients' computed tomography (CT) scans were studied in this work. All the patients were simulated and treated in a supine position using customized thermoplastic masks. The target volumes were CTV_high (high risk target volume) and CTV_low (low risk volume, including lymph nodes). OARs considered were parotids, brainstem, cord, and oral cavity. The volumes of the target and OARs were listed in Table 1. Doses were prescribed in Gy relative biological effectiveness [RBE], assuming an RBE value of 1.1 for protons. The prescribed dose to CTV_high was 70Gy [RBE] in 35 fractions of 2Gy [RBE] per fraction; to CTV_low, in $1.71 \mathrm{~Gy}[\mathrm{RBE}]$ per fraction. The planning goals were as follows: $98 \%$ of the CTVs had to be covered by $100 \%$ of the prescription dose. The similar objectives and constraints for organs OARs were used for both plans.

For beam arrangements, three fields (bilateral oblique directions and one Posterior-Anterior direction) were used for IMPT plan. SPArc plan utilized a full arc trajectory (360 degrees) with a sampling frequency of 2.5 
Table 1 The volume of targets and OARs for fourteen patients. (Unit:cc)

\begin{tabular}{|c|c|c|c|c|c|c|c|}
\hline NO. & CTV high & CTV low/median & Ipsilateral Parotid & Contralateral Parotid & Cord & Brain Stem & Oral Cavity \\
\hline 1 & 141.7 & 204.5 & 38.8 & 39.4 & 16.3 & 12.9 & 74.6 \\
\hline 2 & 418.2 & 202.4 & 23.9 & 12.7 & 18.7 & 24.6 & 81.5 \\
\hline 3 & 92.8 & 172.1 & 19.8 & 12.7 & 16.0 & 29.2 & 190.3 \\
\hline 4 & 173.7 & 400.5 & 53.1 & 57.0 & 12.8 & 19.8 & 136.3 \\
\hline 5 & 234.2 & 240.7 & 29.8 & 30.5 & 33.2 & 30.6 & 109.0 \\
\hline 6 & 127.9 & 283.4 & 43.0 & 41.2 & 8.9 & 7.9 & 55.7 \\
\hline 7 & 106.7 & 119.3 & 20.1 & 23.6 & 25.9 & 7.1 & 61.4 \\
\hline 8 & 124.3 & 324.0 & 43.7 & 41.8 & 14.2 & 20.8 & 127.2 \\
\hline 9 & 174.2 & 394.2 & 20.2 & 27.6 & 49.3 & 36.7 & 52.0 \\
\hline 10 & 238.6 & 200.8 & 30.2 & 35.7 & 19.1 & 28.0 & 44.7 \\
\hline 11 & 65.7 & 374.6 & 22.4 & 26.8 & 17.0 & 28.9 & 95.7 \\
\hline 12 & 100.8 & 286.8 & 33.8 & 31.7 & 29.7 & 19.4 & 59.7 \\
\hline 13 & 97.6 & 166.7 & 32.2 & 39.6 & 30.1 & 15.3 & 49.2 \\
\hline 14 & 58.0 & 225.3 & 18.9 & 18.2 & 31.6 & 13.8 & 35.3 \\
\hline Average & 153.9 & 256.8 & 30.7 & 31.3 & 23.1 & 21.1 & 83.7 \\
\hline Standard Deviation & 94.0 & 89.4 & 10.7 & 12.3 & 10.8 & 9.0 & 43.7 \\
\hline
\end{tabular}

degrees pre-control point [17] without using an RS. Both IMPT and SPArc treatment plans were generated using the RayStation (RaySearch Laboratories AB, Stockholm, Sweden) treatment planning system (TPS) version 6.0 with the same robust optimization parameters: $\pm 3.5 \%$ range and $3 \mathrm{~mm}$ setup uncertainties (a total of 21 worstcase-scenarios), $3 \mathrm{~mm}$ dose grid and 0.02 minimum monitor unit (MU) per spot.

\section{SPArc algorithm}

SPArc is an advanced form of IMPT. Its optimization algorithm was based on the worst-case scenario robust optimization integrated with iterative approaches, including (1) control point re-sampling, (2) control point energy layers re-distribution, and (3) control point energy layers filtration, (4) energy layers re-sampling and (5) spot number reduction. With these approaches, SPArc generates plans that are both robust and delivery efficient, which have the potential to be delivered using the existing clinical proton system [18]. Details of the algorithm are described by Ding et al. in 2016 [17].

\section{Plan quality and robustness evaluation}

The DVHs of both target volumes and OARs were generated on the nominal dose distributions for plan quality evaluation. Clinically significant dosimetric parameters including the maximum dose (D1, the maximum dose covering $1 \%$ volume of a structure) of the brain stem and spinal cord, mean dose of the parotid and, oral cavity were analyzed. Homogeneity Index (HI) was also evaluated based on the Radiation Therapy Oncology Group (RTOG) recommendations, calculated as follows:

$$
H I=\frac{D 5}{D 95}
$$

where $\mathrm{D} x$ is the maximum dose covering $x \%$ volume of a structure.

The conformality index $(\mathrm{CI})$ of target coverage was evaluated as $[19,20]$ :

$$
C I=\frac{T V D p}{T V} \times \frac{T V D p}{V D p}
$$

where $T V D p, T V$, and $V D p$ are the target volume covered by the prescribed dose, target volume, and the volume enclosed by the prescription isodose line respectively.

The integral dose (ID $(\mathrm{Gy} \cdot \mathrm{L})$ ) of radiation delivered to the whole patient body structure or external was defined as:

$$
\mathrm{ID}=\bar{D} \cdot \mathrm{V}
$$

where $\bar{D}$ (Gy) is the mean dose delivered to volume $\mathrm{V}$ (L) (where L - liter). ID formula was employed to calculate and compare the absorbed dose in the patient body [21].

For plan robustness evaluation, the perturbed dose for both SPArc and IMPT plans were generated with $3 \mathrm{~mm}$ isocenter shift in the anterior-posterior, superiorinferior, and right-left directions under nominal proton beam range, with $+3.5 \%$ and $-3.5 \%$ proton beam ranges uncertainties, corresponding to a total of 21 dose distribution scenarios. The DVHs for all the scenarios were plotted for comparisons. The root-mean-square dose (RMSD) for each voxel was calculated as a measure of 
the robustness. The RMSD volume histograms (RVHs) and the Areas Under the RVH Curve (AUC) were compared to quantitatively evaluate the robustness [11, 20, $22,23]$. The smaller the AUC value indicated better plan robustness.

\section{Treatment delivery efficiency estimation}

For delivery efficiency comparison between SPArc and IMPT, total treatment delivery times were estimated based on a 360 gantry with 1 revolution per minute (RPM) gantry rotation speed, $2 \mathrm{~ms}$ spot position switching time, Energy Layer Switching Time (ELST) from $0.1 \mathrm{~s}$ to $5 \mathrm{~s}$ simulating different proton machines in the current market [17].

\section{Potential clinical benefit for parotid glands}

The benefits of SPArc in the clinical setting were estimated using the normal tissue complication probability (NTCP) model. This model was utilized to predict the probability of a reduction in salivary flow to $<25 \%$ of the baseline level at $\leq 6$ months after radiotherapy [24].

We use the NTCP model defined as below $[1,25,26]$ :

$$
\mathrm{NTCP}=\frac{1}{\sqrt{2 \pi}} \int_{-\infty}^{t} e^{-\frac{x^{2}}{2}} d x, t=\frac{\mathrm{MD}-T D_{50}}{m T D_{50}}
$$

Where $M D$ is the mean organ dose, $T D_{50}$ is the uniform dose given to the entire organ volume that results in $50 \%$ complication risk, $\mathrm{m}$ is a measure of the slope of the sigmoid curve represented by the integral of the normal distribution. ' $m$ ' and ' $T D_{50}$ ' were set as $0.53,31.4 \mathrm{~Gy}$, respectively using combined organ analysis of the Chao et al. data [1]. The mean dose was given to both parotid glands in this study.

\section{Statistic analysis}

The difference of all parameters between IMPT and SPArc was assessed with a paired, 2-tailed nonparametric Wilcoxon signed-rank test using SPSS 21.0 software (International Business Machines, Armonk, New York) and $p$ values less than 0.05 were considered statistically significant.

\section{Results}

\section{Plan Dosimetric comparison}

The study showed that the SPArc treatment plans achieved better conformal dose distribution compared to the IMPT. Figure 1 shows a representative CT slice of a bilateral HNC case (patient \#8) with coronal and sagittal view dose comparison for SPArc and IMPT as well as the corresponding DVHs, respectively. The average $\mathrm{HI}$ and CI of SPArc plan are $1.04 \pm 0.01$ and $0.70 \pm 0.06$ for CTV_high, $1.07 \pm 0.04$ and $0.54 \pm 0.09$ for CTV_low, which are significantly improved in comparison with
IMPT (CTV_high: $\mathrm{HI}=1.05 \pm 0.01, p=0.005, \mathrm{CI}=0.67 \pm$ $0.07, P=0.006$; CTV_low: $\mathrm{HI}=1.09 \pm 0.05, p=0.003, \mathrm{CI}=$ $0.51 \pm 0.10, p=0.017)$. Table 2 listed the dosimetric metrics of fourteen patients SPArc and IMPT treatment plans. SPArc had demonstrated significant dosimetric improvements over IMPT throughout all critical organs analyzed. More specifically, SPArc decreased the mean dose to the ipsilateral parotid, contralateral parotid, and oral cavity by $25.8 \%(p=0.001), 20.8 \%(\mathrm{p}=0.001)$ and $20.3 \%(\mathrm{p}=0.001)$ respectively. Moreover, the D1 of cord and brain stem was decreased by SPArc (20.8\%, $p=0.009$, and $10.7 \%, p=0.048)$. Also, SPArc plans decreased the average integral dose from $147.0 \pm 33.8 \mathrm{~Gy} \cdot \mathrm{L}$ to $121.8 \pm 29.7 \mathrm{~Gy}$. L, by $17.2 \%$ $(\mathrm{p}=0.001)$ compared to IMPT plans. SPArc improved the V3Gy of external from $5137.8 \pm 1331.7 \mathrm{cc}$ to $4710.8 \pm 1328.4 \mathrm{cc}(p=0.008)$ as well.

\section{Plan robustness evaluation}

The robustness of all the treatment plans was evaluated with 21 worst-case-scenarios. The perturbed DVHs for patient \#8 are shown in Fig. 2 (a) target coverage, and Fig. 2 (b) OARs sparing. Both SPArc and IMPT plans could achieve adequate target coverage, with at least 98\% of CTV_high receiving the prescription dose. Compared to IMPT, the SPArc plan significantly reduced the average AUC value for the cord from 1.1 $\pm 0.4 \mathrm{~Gy}$ [RBE] to $0.7 \pm 0.3 G y[R B E](p=0.001)$, for brain stem from $0.9 \pm 0.7 \mathrm{~Gy}[\mathrm{RBE}]$ to $0.7 \pm 0.7 \mathrm{~Gy}[\mathrm{RBE}] \quad(p=0.019)$, for contralateral parotid from $2.5 \pm 0.5 \mathrm{cGy}[\mathrm{RBE}]$ to $2.2 \pm$ $0.6 \mathrm{~Gy}[\mathrm{RBE}](p=0.022)$ and for ipsilateral parotid from $3.1 \pm 0.7 \mathrm{cGy}[\mathrm{RBE}]$ to $2.8 \pm 0.6 \mathrm{~Gy}[\mathrm{RBE}] \quad(p=0.004)$. SPArc treatment plan is comparable to IMPT plan in terms of robustness for CTV_high, CTV_low, and oral cavity. Moreover, Fig. 3 (a) showed the corresponding average mean AUC index of target volumes and OARs of the fourteen cases with their corresponding $\mathrm{p}$ values. Moreover, an example of the rootmean-square dose (RMSD) volume histograms of SPArc (solid line) and IMPT for patient \#8 is given in Fig. 3 (b).

\section{Total estimated treatment delivery time}

Figure 4(a) shows the estimated delivery time per fraction for both SPArc and IMPT plans simulating proton systems with various ELST. For the proton centers with ELST equaling to $5 \mathrm{~s}$, the estimated delivery time is significantly longer for SPArc $(1294.9 \pm 106.7 \mathrm{~s}$ vs. $901.7 \pm$ $74.8 \mathrm{~s} p<0.001$ ), however, the difference of treatment delivery time decreases rapidly as the ELST shortens. The delivery time for both modalities are comparable at the $\quad E L S T=0.5 \mathrm{~s}, \quad 366.0 \pm 58.5 \mathrm{~s}$ (SPArc) $\quad$ vs374.6 \pm 49.7 $\mathrm{s}(\mathrm{IMPT})(p=0.124)$, and it is even significantly shorter in SPArc plan at ELST $\leq 0.2 \mathrm{~s}, 303.3 \pm 57.3 \mathrm{~s}$ (SPArc) vs 


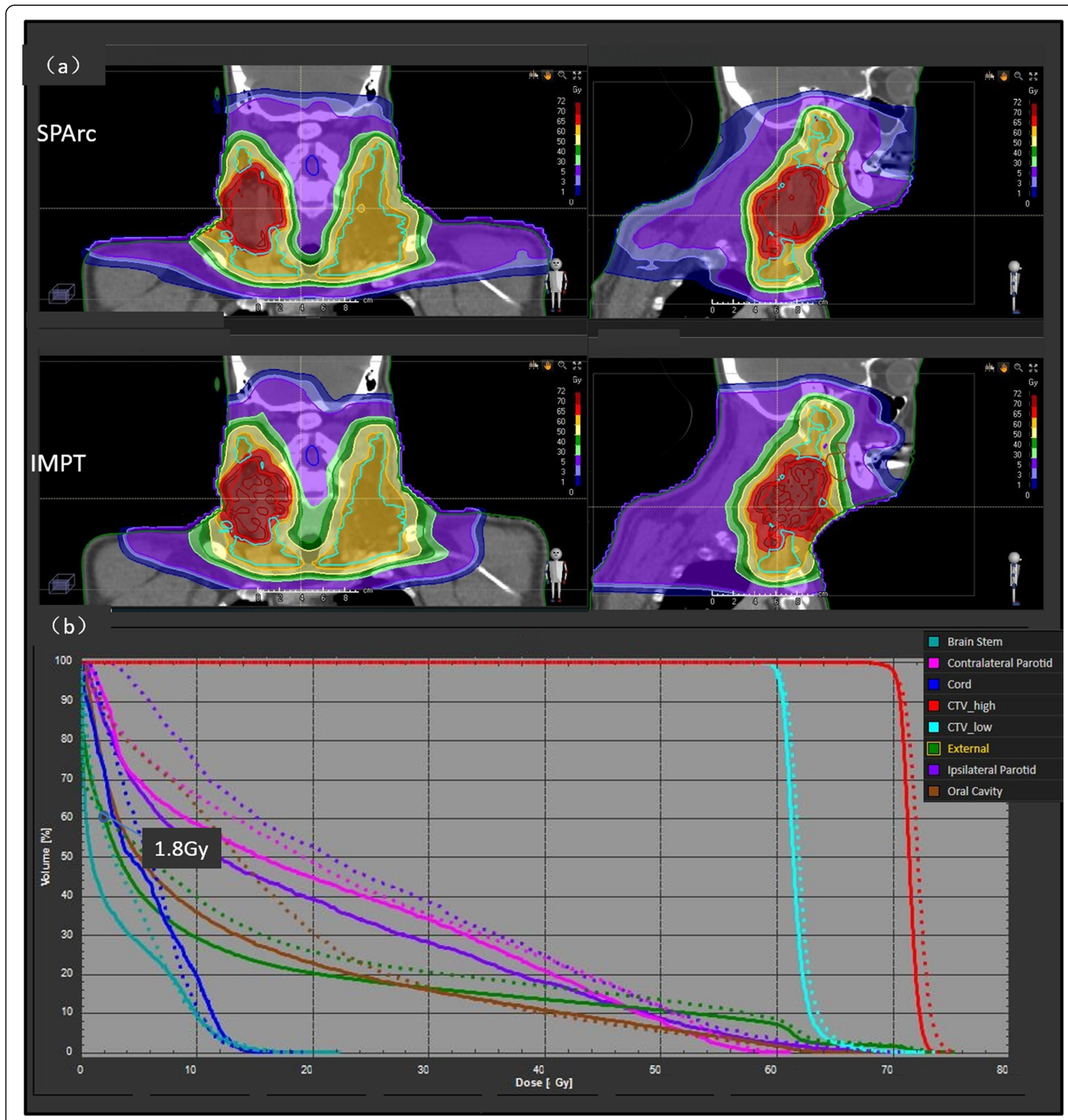

Fig. 1 The comparison of (a) dose distributions of SPArc and IMPT plans for patient 8 (b) the corresponding DVH as an example (SPArc:Solid line, IMPT:dash line). The dot as $1.8 \mathrm{~Gy}$ represents the intersection of external between SPArc and IMPT plan

$339.7 \pm 48.1 \mathrm{~s} \quad(\mathrm{IMPT}) \quad(\mathrm{p}=0.004) \quad$ with $\quad$ ELST $=0.2 \mathrm{~s}$, $283.4 \pm 56.2 \mathrm{~s}(\mathrm{SPArc})$ vs $328.0 \pm 47.6 \mathrm{~s}(\mathrm{IMPT})(p=0.002)$ with ELST $=0.1 \mathrm{~s}$.

The average calculation time required to generate a SPArc plan for bilateral HNC is between 6 to $8 \mathrm{~h}$, depending on the size of the target volume. This simulation was performed on a 64-bit workstation with an Intel Quad-Core processor (TM i5-4590 CPU @ 3.30 $\mathrm{GHz}$ ) and 64 GB RAM.

\section{Potential clinical benefit for parotid glands}

The results showed that there was a potential clinical benefit in terms of estimated salivary flow dysfunction based on mean NTCP values while comparing SPArc to IMPT. The estimated reduction in NTCP varied widely among patients (Fig. 4(b)). As compared with IMPT, SPArc decreased the mean probability of salivary flow dysfunction from $36 \pm 9$ to $25 \% \pm 10 \%(\mathrm{p}=0.001)$ after radiotherapy. 
Table 2 The average dosimetric characteristic for the fourteen patients

\begin{tabular}{|c|c|c|c|c|}
\hline Structure & Value & SPArC & IMPT & pValue \\
\hline \multirow[t]{4}{*}{ CTV_high } & D95(Gy) [RBE] & $70.8 \pm 0.2$ & $70.7 \pm 0.2$ & 0.975 \\
\hline & D5(Gy) [RBE] & $73.6 \pm 0.8$ & $74.1 \pm 0.7$ & 0.011 \\
\hline & $\mathrm{HI}$ & $1.04 \pm 0.01$ & $1.05 \pm 0.01$ & 0.005 \\
\hline & $\mathrm{Cl}$ & $0.70 \pm 0.06$ & $0.67 \pm 0.07$ & 0.006 \\
\hline \multirow[t]{4}{*}{ CTV_low } & D95(Gy) [RBE] & $60.8 \pm 0.4$ & $60.8 \pm 0.4$ & 0.777 \\
\hline & D5(Gy) [RBE] & $64.6 \pm 1.0$ & $65.7 \pm 1.7$ & 0.003 \\
\hline & $\mathrm{HI}$ & $1.07 \pm 0.04$ & $1.09 \pm 0.05$ & 0.003 \\
\hline & $\mathrm{Cl}$ & $0.54 \pm 0.09$ & $0.51 \pm 0.10$ & 0.017 \\
\hline Cord & D1(Gy) [RBE] & $17.2 \pm 7.0$ & $21.7 \pm 7.9$ & 0.009 \\
\hline Brain Stem & D1(Gy) [RBE] & $14.2 \pm 11.1$ & $15.9 \pm 11.3$ & 0.048 \\
\hline Contralateral Parotid & Mean Dose (Gy) [RBE] & $17.9 \pm 6.4$ & $22.6 \pm 5.3$ & 0.001 \\
\hline Ipsilateral Parotid & Mean Dose (Gy) [RBE] & $21.1 \pm 7.4$ & $28.4 \pm 6.2$ & 0.001 \\
\hline Oral Cavity & Mean Dose (Gy) [RBE] & $21.9 \pm 9.7$ & $27.5 \pm 9.4$ & 0.001 \\
\hline External & ID (Gy · L) & $121.8 \pm 29.7$ & $147.0 \pm 33.8$ & 0.001 \\
\hline V1Gy & $\mathrm{CC}$ & $6186.9 \pm 1800.1$ & $5852.7 \pm 1543.5$ & 0.011 \\
\hline V3Gy & $\mathrm{CC}$ & $4710.8 \pm 1328.4$ & $5137.8 \pm 1331.7$ & 0.008 \\
\hline
\end{tabular}

Abbreviations: $H I$ (homogeneity index), RBE relative biological effectiveness, $D x$ the maximum dose covering $x \%$ volume of a structure, ID the integral dose, $V_{x}$ the volume of a structure received $x$ Gy [RBE]

\section{Discussion}

Radiation-induced side effects, including xerostomia and dysphagia, have significant impacts on the quality of life of HNC patients [27-29]. Dosimetric improvements could potentially improve the treatment outcome by reducing acute and late toxicities. Recent studies have shown improved IMPT robustness associated with the increase of treatment fields while decreasing OARs sparing [12, 30, 31]. However, such approaches using numerous static beam angles were considered impractical due to the prolonged treatment delivery time, especially in a multi-room proton center where significant time will be spent in the room-switching time/waiting time [32]. Since the introduction of the SPArc technique in 2016 [17], there have been efforts to investigate the potential dosimetric improvements via SPArc in different disease sites $[11,20,23]$. Our work is the first comprehensive study to exploit the potential advantage of utilizing SPArc for bilateral HNC radiotherapy in terms of OARs sparing, target robust coverage, and treatment delivery efficiency. These results not only
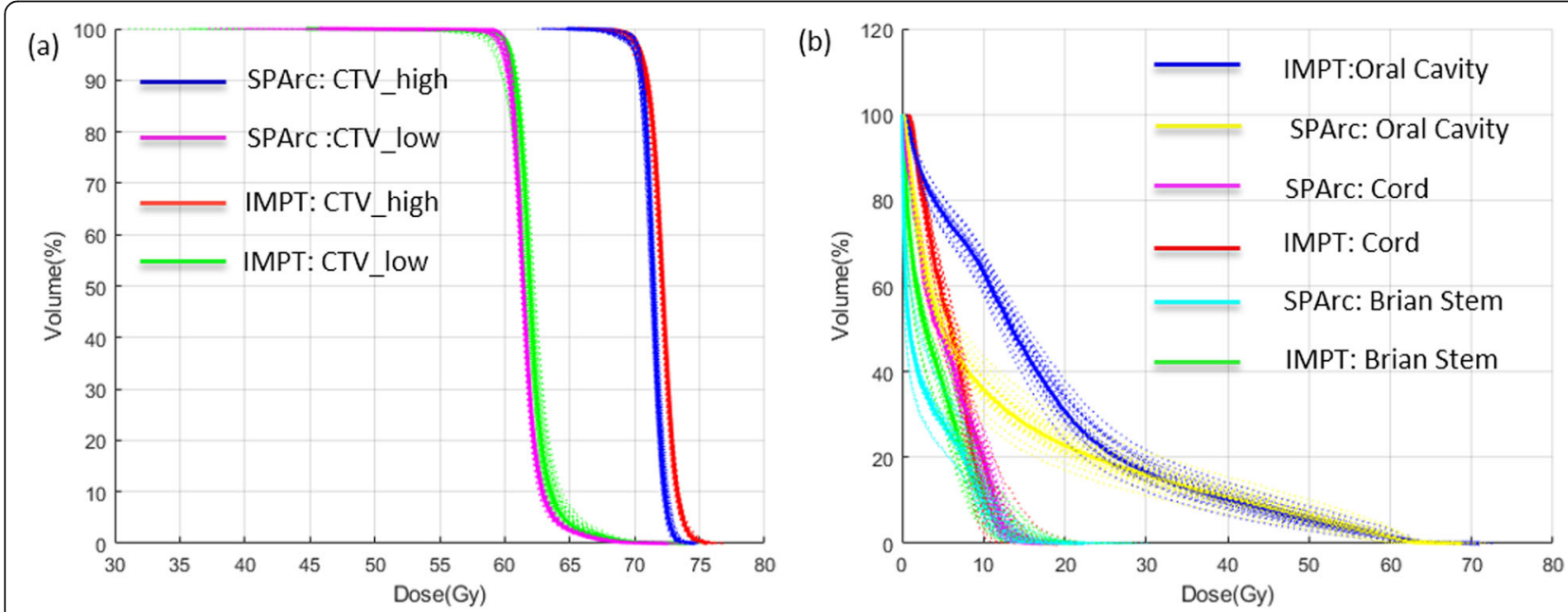

Fig. 2 The DVHs of nominal position (solid line) and 20 scenarios of uncertainties (dashed line) for (a) target and (b) OARs for patient \#8 

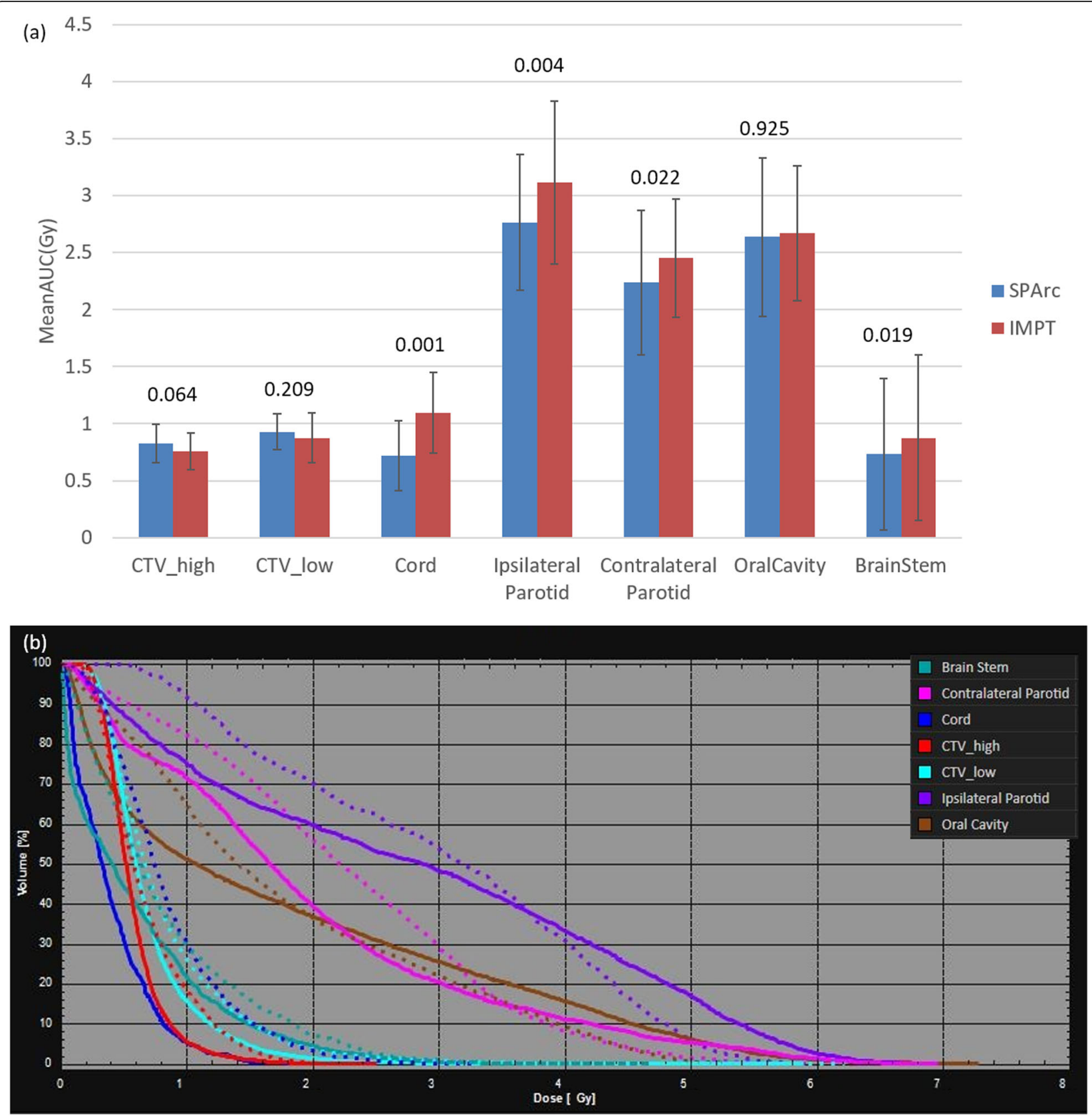

Fig. 3 a The average areas under the RVH curve (AUC) for fourteen patients with $p$-values on the top of the columns. $\mathbf{b}$ An example of the rootmean-square dose (RMSD) volume histograms of SPArc (solid line) and IMPT (dashed line) for patient \# 8

consolidated the findings from previous publications regarding further improving the plan quality [33, 34] but also introduced a novel treatment technique that could shorten the proton treatment time and simplify the proton clinical treatment workflow without using an RS through a dynamic arc trajectory.

At the current stage, the SPArc technique is still a concept that is under development towards the clinical product. One of the biggest challenges is the capability of delivering numerous spots with a significantly lower
MU weighting compared to the traditional 3-field IMPT technique. Figure 5 showed an example of the spot weighting distribution comparison between the 3-field IMPT and SPArc plan for the HNC patient \#8. The plan delivery accuracy and efficiency might be sensitive to the design of the beamline, ion chambers, and tolerance threshold of beam position and profile, which varies among different vendors or models of proton systems. The results from these QA experiments demonstrate the feasibility and compatibility of the SPArc delivery 


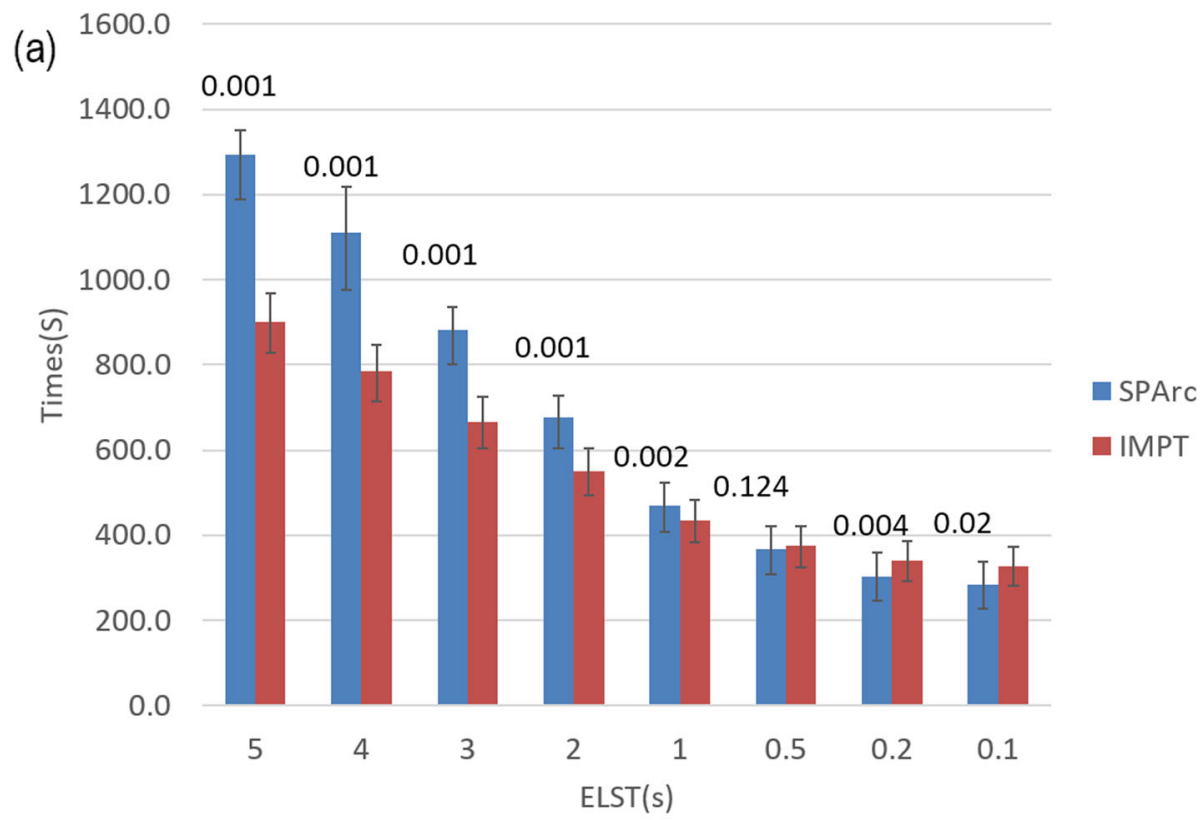

(b)

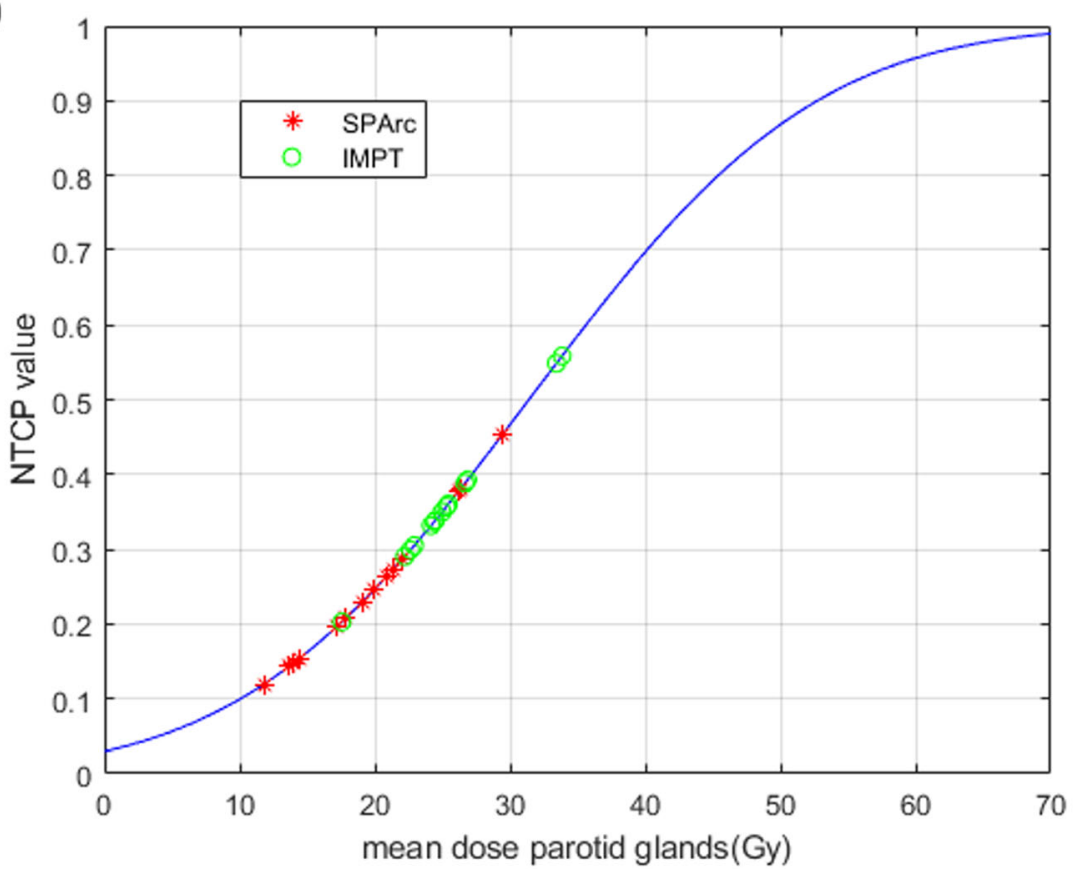

Fig. 4 a The average estimated delivery time per fraction varies along with the ELST with p-values on the top of the columns. $\mathbf{b}$ Normal tissue complication probability (NTCP) model for parotid salivary flow (solid curve). The NTCP value denotes the probability of a reduction in salivary flow to $<25 \%$ of the pretreatment flow at $\leq 6$ months after radiotherapy

on an existing proton therapy system. However, we did observe one or two beams pauses/check on some SPArc plans treatment delivery, which was due to the noise of the ion chamber in delivering such highly modulated spots with low MU weightings. As a result, it is critical to understand the difference in the machine-specific limitations while generating the SPArc plan and arc delivery sequence. The next phase of development is to design a new control software and hardware which could deliver such proposed dynamic arc therapy in a safe, robust, accurate, and efficient way.

The use of RS in treating superficial cancer targets such as bilateral HNC complicates the clinical workflow in terms of the clearance check, especially near the shoulder region [12]. It also introduces secondary proton 


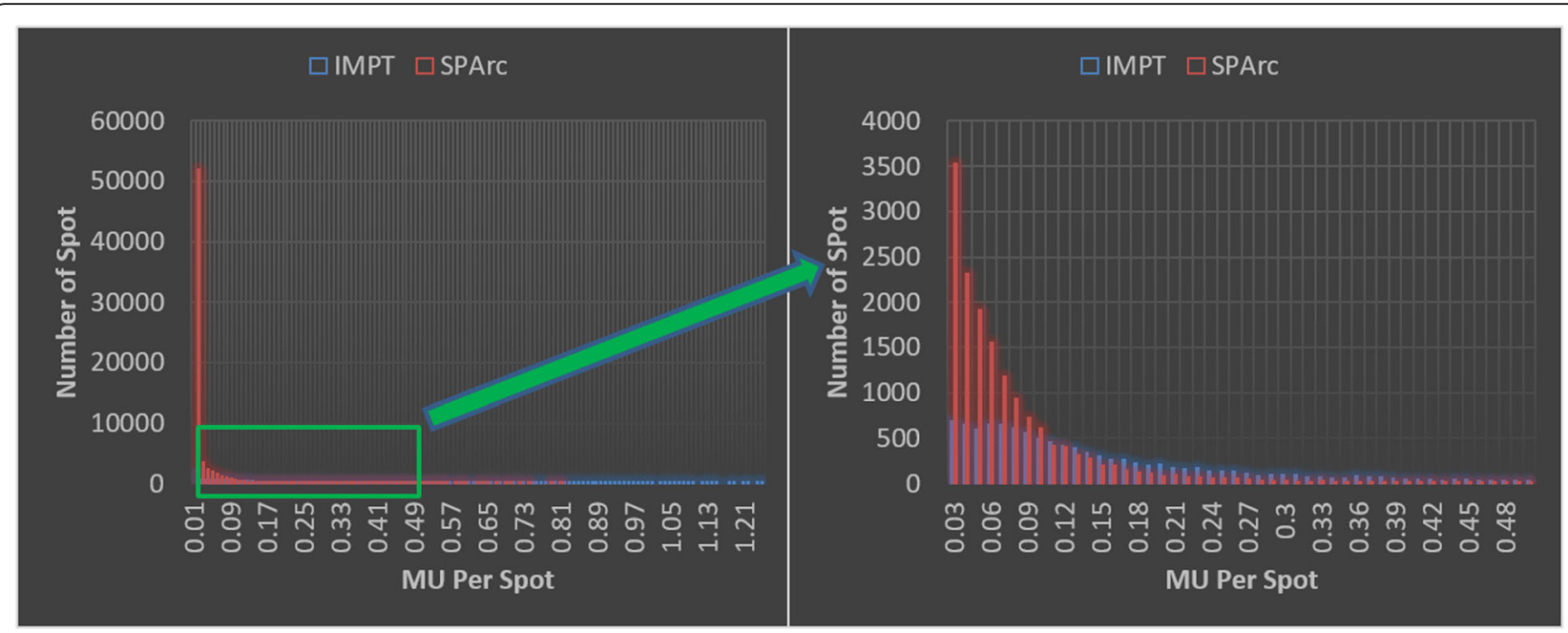

Fig. 5 Spot vs MU weighting Histogram on an example patient case \#8

scattering from the RS, which increases spot size when entering the patient's body and thus degrades the treatment plan quality due to the larger lateral penumbra [35-37]. There are numerous publications to address the clearance issues as well as proton beam modeling and dosimetric plan quality challenges of using RS [38, 39]. This study demonstrated that SPArc does not need to use RS while providing a superior dosimetric plan quality, simplifies the clinical workflow with a practically achievable treatment delivery time compared to the current standard-of-care IMPT.

Last but not the least, a more substantial volume of low dose bath is one of the biggest concerns in the proton arc therapy approach where the beam directions are coming from an arc(s) trajectory. The advantage of traditional proton beam therapy using limited beam angles can spare the normal tissue volume away from the target with almost zero doses. This feature is critical to the pediatric patients' treatment, as most of them are expected to live much longer. Thus, it is crucial to reduce the chance of radiation-induced secondary malignancy by choosing the most appropriated beam angles [40, 41]. Our study showed that SPArc therapy has a higher V1Gy $(p=0.011)$ but a lower V3Gy $(p=0.008)$ in the treatment of bilateral HNC compared to the IMPT (Table 2). Although the 1Gy volume is higher, it is interesting to find that the total integral dose in bilateral HNC treatment is lower than IMPT which agrees with the findings in the lung and prostate studies as well $[11,20]$. At the current stage, the clinical significance of low dose bath or integral dose in proton therapy is not well known yet, such concerns of radiation-induced secondary malignancy may limit the usage of proton arc technique in pediatric patients' treatment. Based on this study, we are able to conclude that the significant dosimetric advantage of
SPArc therapy is to offer a better dose conformity and a better sparing the high or median-dose volume which is critical to the disease sites where the OARs adjunct to the target volume such as HNC where the average age is between 50 and 70 years old [42]. Such a feature could allow proton beam therapy to reduce acute radiation toxicity furthermore.

\section{Conclusions}

SPArc is a robust and delivery-efficient proton arc therapy technique that could potentially be implemented into routine clinical practice to further improve the treatment outcomes in $\mathrm{HNC}$ patients.

\section{Abbreviations \\ 3DCRT: 3-dimensional conformal radiation therapy; AUC: Areas Under the RVH Curve; CT: computed tomography; DVH: dose volume histogram; ELST: Energy Layer Switching Time; HI: Homogeneity Index; HNC: head and neck cancer; IMPT: Intensity Modulated Proton Therapy; MU: Monitor unit; NTCP: Normal tissue complication probability; OAR: Organs-at-risk; PBS: Pencil Beam Scanning; RBE: Relative biological effectiveness; RMSD: Root-mean- square dose; RS: Range shifter; RT: Radiotherapy; RTOG: Radiation Therapy Oncology Group; RVH: RMSD volume histograms; SPArc: Spot-scanning Proton Arc therapy; TPS: Treatment planning system; VMAT: Volumetric- modulated arc therapy}

\section{Acknowledgements}

This study is supported by research funding from IBA and Beaumont Health Herb and Betty Fisher Research Seed Grant Award.

\section{Authors' contributions}

GL contribute to the acquisition, analysis and interpretation of data and draft and design the paper. $X \mathrm{~L}$ and $\mathrm{WZ}$ contribute to revising the paper and providing clinical inputs; $A Q, D Y$ and $S Z$ provide imaging acquisition and support; PK contribute to the statistical analysis and provided clinical inputs. $X D$, contribute to the design of the study, revise the draft and lead the research direction. All authors read and approved the final manuscript.

\section{Funding}

This study was supported by the research funding from lon Beam Application Inc. (IBA, Belgium) and Beaumont Health Herb and Betty Fisher Research Seed Grant Award. 


\section{Availability of data and materials}

All data generated or analyzed during this study are included in this published article. Additional information is available from the corresponding author on reasonable request.

\section{Ethics approval and consent to participate}

The patient data used in this study is approved by Beaumont institutional review board.

\section{Consent for publication}

Not applicable.

\section{Competing interests}

Xuanfeng Ding, Xiaoqiang Li and Di Yan have a patent related to the Particle Arc Therapy (WO2017156419).

\section{Author details}

'Cancer Center, Union Hospital, Tongji Medical College, Huazhong University of Science and Technology, Wuhan 430023, China. ${ }^{2}$ Department of Radiation Oncology, Beaumont Health System, Royal Oak, MI 48074, USA. ${ }^{3}$ School of Physics and Technology, Wuhan University, Hubei, Wuhan 430072, China.

Received: 30 July 2019 Accepted: 20 January 2020

Published online: 30 January 2020

\section{References}

1. Chao KSC, Deasy JO, Markman J, Haynie J, Perez CA, Purdy JA, et al. A prospective study of salivary function sparing in patients with head-andneck cancers receiving intensity-modulated or three-dimensional radiation therapy: initial results. Int J Radiat Oncol. 2001;49:907-16.

2. Dirix P, Vanstraelen B, Jorissen M, Vander Poorten V, Nuyts S. Intensitymodulated radiotherapy for Sinonasal Cancer: improved outcome compared to conventional radiotherapy. Int J Radiat Oncol. 2010;78:9981004.

3. Eisbruch A, Harris J, Garden AS, Chao CKS, Straube W, Harari PM, et al. Multiinstitutional trial of accelerated Hypofractionated intensity-modulated radiation therapy for early-stage Oropharyngeal Cancer (RTOG 00-22). Int J Radiat Oncol. 2010;76:1333-8.

4. Cozzi L, Fogliata A, Lomax A, Bolsi A. A treatment planning comparison of 3D conformal therapy, intensity modulated photon therapy and proton therapy for treatment of advanced head and neck tumours. Radiother Oncol. 2001;61:287-97.

5. Steneker M, Lomax A, Schneider U. Intensity modulated photon and proton therapy for the treatment of head and neck tumors. Radiother Oncol. 2006; 80:263-7.

6. van de Water TA, Lomax AJ, Bijl HP, de Jong ME, Schilstra C, Hug EB, et al. Potential benefits of scanned intensity-modulated proton therapy versus advanced photon therapy with regard to sparing of the salivary glands in Oropharyngeal Cancer. Int J Radiat Oncol. 2011;79:1216-24.

7. Lomax AJ, Pedroni E, Rutz HP, Goitein G. The clinical potential of intensity modulated proton therapy. Z Für Med Phys. 2004;14:147-52.

8. Lomax AJ. Intensity modulated proton therapy and its sensitivity to treatment uncertainties 2: the potential effects of inter-fraction and interfield motions. Phys Med Biol. 2008;53:1043-56.

9. Lomax AJ. Intensity modulated proton therapy and its sensitivity to treatment uncertainties 1: the potential effects of calculational uncertainties. Phys Med Biol. 2008;53:1027-42.

10. Cubillos-Mesías M, Baumann M, Troost EGC, Lohaus F, Löck S, Richter C, et al. Impact of robust treatment planning on single- and multi-field optimized plans for proton beam therapy of unilateral head and neck target volumes. Radiat Oncol [Internet]. 2017 [cited 2018 Jun 26];12. Available from: https://ro-journal.biomedcentral.com/articles/10.1186/s13014-0170931-8

11. Ding X, Li X, Qin A, Zhou J, Yan D, Stevens C, et al. Have we reached proton beam therapy dosimetric limitations? - a novel robust, delivery-efficient and continuous spot-scanning proton arc (SPArc) therapy is to improve the dosimetric outcome in treating prostate cancer. Acta Oncol. 2018;57:435-7.

12. Ding X, Li X, Qin A, Zhou J, Yan D, Chen P, et al. Redefine the role of range shifter in treating bilateral head and neck cancer in the era of Intensity Modulated Proton Therapy. J Appl Clin Med Phys [Internet]. 2018 [cited 2018 Jul 23]; Available from: http://doi.wiley.com/10.1002/acm2.12416
13. Moignier A, Gelover E, Wang D, Smith B, Flynn R, Kirk M, et al. Improving head and neck Cancer treatments using dynamic collimation in spot scanning proton therapy. Int J Part Ther. 2016;2:544-54.

14. Wang D, Dirksen B, Hyer DE, Buatti JM, Sheybani A, Dinges E, et al. Impact of spot size on plan quality of spot scanning proton radiosurgery for peripheral brain lesions: spot scanning proton radiosurgery. Med Phys. 2014;41:121705.

15. Dijkema T, Terhaard CHJ, Roesink JM, Braam PM, van Gils CH, Moerland MA, et al. Large cohort dose-volume response analysis of parotid gland function after radiotherapy: intensity-modulated versus conventional radiotherapy. Int J Radiat Oncol. 2008;72:1101-9.

16. Paganetti $H$, Yu CX, Orton CG. Photon radiotherapy has reached its limit in terms of catching up dosimetrically with proton therapy: point/ counterpoint. Med Phys. 2016;43:4470-2.

17. Ding X, Li X, Zhang JM, Kabolizadeh P, Stevens C, Yan D. Spot-Scanning Proton Arc (SPArc) Therapy: The First Robust and Delivery-Efficient SpotScanning Proton Arc Therapy. Int J Radiat Oncol Biol Phys. 2016;96:1107-16.

18. Li X, Liu G, Janssens G, De Wilde O, Bossier V, Lerot X, et al. The first prototype of spot-scanning proton arc treatment delivery. Radiother Oncol. 2019;137:130-6.

19. Quan EM, Li X, Li Y, Wang X, Kudchadker RJ, Johnson JL, et al. A comprehensive comparison of IMRT and VMAT plan quality for prostate Cancer treatment. Int J Radiat Oncol. 2012;83:1169-78.

20. Li X, Kabolizadeh P, Yan D, Qin A, Zhou J, Hong Y, et al. Improve dosimetric outcome in stage III non-small-cell lung cancer treatment using spotscanning proton arc (SPArc) therapy. Radiat Oncol [Internet]. 2018 [cited 2018 Jun 17];13. Available from: https://ro-journal.biomedcentral.com/ articles/10.1186/s13014-018-0981-6

21. Aoyama H, Westerly DC, Mackie TR, Olivera GH, Bentzen SM, Patel RR, et al. Integral radiation dose to normal structures with conformal external beam radiation. Int J Radiat Oncol. 2006;64:962-7.

22. Liu W, Frank SJ, Li X, Li Y, Park PC, Dong L, et al. Effectiveness of robust optimization in intensity-modulated proton therapy planning for head and neck cancers: robust optimization for IMPT for H\&N cancer. Med Phys. 2013;40:051711.

23. Ding X, Zhou J, Li X, Blas K, Liu G, Wang Y, et al. Improving dosimetric outcome for hippocampus and cochlea sparing whole brain radiotherapy using spot-scanning proton arc therapy. Acta Oncol. 2019:0:1-8.

24. Semenenko VA, Li XA. Lyman-Kutcher-Burman NTCP model parameters for radiation pneumonitis and xerostomia based on combined analysis of published clinical data. Phys Med Biol. 2008;53:737-55.

25. Kutcher GJ, Burman C. Calculation of complication probability factors for non-uniform normal tissue irradiation: the effective volume method Gerald. Int J Radiat Oncol. 1989;16:1623-30.

26. Lyman JT. Complication probability as assessed from dose-volume histograms. Radiat Res. 1985:104:13-9.

27. van Dijk LV, Steenbakkers RJHM, ten Haken B, van der Laan HP, van 't Veld AA, Langendijk JA, et al. Robust Intensity Modulated Proton Therapy (IMPT) Increases Estimated Clinical Benefit in Head and Neck Cancer Patients. PLOS ONE. 2016;11:e0152477.

28. Dirix $\mathrm{P}$, Nuyts $\mathrm{S}$. Evidence-based organ-sparing radiotherapy in head and neck cancer. Lancet Oncol. 2010;11:85-91.

29. Langendijk JA, Doornaert P, Verdonck-de Leeuw IM, Leemans CR, Aaronson NK, Slotman BJ. Impact of late treatment-related toxicity on quality of life among patients with head and neck Cancer treated with radiotherapy. J Clin Oncol. 2008;26:3770-6.

30. Pflugfelder D, Wilkens JJ, Oelfke U. Worst case optimization: a method to account for uncertainties in the optimization of intensity modulated proton therapy. Phys Med Biol. 2008;53:1689-700.

31. Kraan AC, van de Water S, Teguh DN, Al-Mamgani A, Madden T, Kooy HM, et al. Dose uncertainties in IMPT for Oropharyngeal Cancer in the presence of anatomical, range, and setup errors. Int J Radiat Oncol. 2013;87:888-96.

32. Aitkenhead AH, Bugg D, Rowbottom CG, Smith E, Mackay RI. Modelling the throughput capacity of a single-accelerator multitreatment room proton therapy Centre. Br J Radiol. 2012;85:e1263-72.

33. van de Water TA, Bijl HP, Schilstra C, Pijls-Johannesma M, Langendijk JA. The potential benefit of radiotherapy with protons in head and neck Cancer with respect to Normal tissue sparing: a systematic review of literature. Oncologist. 2011;16:366-77.

34. Kainz K, Firat S, Wilson JF, Schultz C, Siker M, Wang A, et al. Comparing the quality of passively-scattered proton and photon tomotherapy plans for brain and head and neck disease sites. Phys Med Biol. 2015;60:2167-77. 
35. Matysiak W, Yeung D, Slopsema R, Li Z. Evaluation of the range shifter model for proton pencil-beam scanning for the eclipse v.11 treatment planning system. J Appl Clin Med Phys. 2016;17:391-404.

36. Shen J, Liu W, Anand A, Stoker JB, Ding X, Fatyga M, et al. Impact of range shifter material on proton pencil beam spot characteristics. Med Phys. 2015; 42:1335-40.

37. Schaffner B. Proton dose calculation based on in-air fluence measurements. Phys Med Biol. 2008:53:1545-62.

38. Fracchiolla F, Fellin F, Innocenzi M, Lipparini M, Lorentini S, Widesott L, et al. A pre-absorber optimization technique for pencil beam scanning proton therapy treatments. Phys Med. 2019;57:145-52.

39. Zhang Y, Kerr MD, Guan F, Hartman J, Jiang B, Sahoo N, et al. Dose calculation for spot scanning proton therapy with the application of a range shifter. Biomed Phys Eng Express. 2017;3:035019.

40. Merchant TE. Proton beam therapy in pediatric oncology. Cancer J. 2009:15:8.

41. Mizumoto M, Oshiro Y, Yamamoto T, Kohzuki H, Sakurai H. Proton Beam Therapy for Pediatric Brain Tumor. Neurol Med Chir (Tokyo). 2017;57:343-55.

42. Stoyanov GS, Kitanova M, Dzhenkov DL, Ghenev P, Sapundzhiev N. Demographics of Head and Neck Cancer Patients: A Single Institution Experience. Cureus [Internet]. [cited 2019 Mar 23];9. Available from: https:/ www.ncbi.nlm.nih.gov/pmc/articles/PMC5580971/

\section{Publisher's Note}

Springer Nature remains neutral with regard to jurisdictional claims in published maps and institutional affiliations.

Ready to submit your research? Choose BMC and benefit from:

- fast, convenient online submission

- thorough peer review by experienced researchers in your field

- rapid publication on acceptance

- support for research data, including large and complex data types

- gold Open Access which fosters wider collaboration and increased citations

- maximum visibility for your research: over $100 \mathrm{M}$ website views per year

At $\mathrm{BMC}$, research is always in progress.

Learn more biomedcentral.com/submissions 